\title{
Gastrointestinal side effects of cancer treatment: are we making any progress?
}

\author{
Authors: Anum Faisal, Waqaar Baber, Zain Rana and Jervoise Andreyev
}

\section{Introduction}

In 2012, the British Society of Gastroenterology, the Association of Coloproctology of Great Britain and Ireland, the Association of Upper Gastrointestinal Surgeons, the College of Radiologists and Macmillan Cancer Support published guidance on the optimal management of acute and chronic gastrointestinal (GI) toxicities of cancer treatments. ${ }^{1}$ The ORBIT trial, ${ }^{2}$ hailed as a landmark study, ${ }^{3}$ showed that targeted interventions, based on the principles in the published guidance delivered by a nurse or a gastroenterologist, can make a significant difference to those with chronic toxicity.

The largest single group of people at risk of moderate/severe long-term side effects are those treated for colorectal cancer. Follow-up after colorectal cancer treatment is increasingly devolved to junior or paramedical staff and historically has largely focused on possible recurrence. This study investigated how toxicity after colorectal cancer is assessed and managed in a large district hospital.

\section{Methods}

Outpatient clinic letters and inpatient discharge letters of a random selection of patients diagnosed with a new primary colorectal cancer over 1 year, treated with curative intent and with at least one follow-up appointment were audited retrospectively.

\section{Results}

A total of 462 patients were discussed at the colorectal MDT in 2017. Notes of 252 patients, $54 \%$ men $(n=136)$ were scrutinised. Of these, $54 \%$ ( $n=145$ age range $26-98$ years) were treated with curative intent. The site of the primary cancer was anus $1 \%(n=2)$, rectum $34 \%(n=49)$, sigmoid $16 \%(n=23)$, left colon $5 \%(n=7)$, transverse $8 \%(n=11)$, right colon $35 \%(n=51)$ and appendix $1 \%$ $(n=2) .79 \%(n=114)$ had a 1-year follow-up.

$65 \%(n=94)$ were treated with surgery alone. $26 \%(n=37)$ underwent surgery and adjuvant therapy (chemotherapy and/ or radiotherapy). $5 \%(n=8)$ had surgery and neoadjuvant therapy (chemotherapy and/or radiotherapy). 3\% had chemotherapy and radiotherapy but no surgery $(n=5)$ and $1 \%(n=1)$ were treated with polypectomy alone.
$19 \%$ were readmitted with complications of surgery $(n=28)-$ mean length of stay, 10 days. Only $50 \%(n=73)$ of follow-up letters documented a conversation about side effects; GI 39\% ( $n=56)$ psychological $6 \%(n=8)$; sexual $1 \%(n=1)$; tiredness $12 \%(n=17)$; quality of life $8 \%(n=11)$; urinary incontinence $8 \%(n=12)$.

GI problems included frequency, urgency, loss of appetite, abdominal pain, weight loss, diarrhoea, rectal bleeding, faecal incontinence and nocturnal defecation. Only $22 \%$ of patients $(n=16)$ were offered investigations other than to rule out recurrent cancer, $41 \%(n=30)$ were offered advice and only $22 \%(n=16)$ were offered follow up to see if this advice helped.

\section{Conclusions}

Survival after colorectal cancer is improving rapidly. However, it is associated with frequent and predictable side effects. In our busy hospital trust, there is little evidence of systematic enquiry about side effects of cancer treatment or appropriate investigation and effective treatments being offered at follow-up. The lessons learnt during the UK's National Cancer Survivorship Initiative launched in 2007 and jointly run by the Department of Health and Macmillan Cancer Support, ${ }^{4}$ have not impacted clinical practice.

\section{References}

1 Andreyev HJN, Davidson SE, Gillespie C, Allum WH, Swarbrick E. Practice guidance on the management of acute and chronic gastrointestinal problems arising as a result of treatment for cancer. Gut 2012:61:179-92.

2 Andreyev HJN, Benton BE, Lalji A, Norton C, Mohammed K, Gage H et al. Algorithm-based management of patients with gastrointestinal symptoms in patients after pelvic radiation treatment (ORBIT): a randomised controlled trial. Lancet 2013;382:2084-92.

3 Denham JW, Hauer-Jensen M. Radiation induced bowel injury: a neglected problem. Lancet 2013;382:2046-7.

4 Macmillan Cancer Support. Throwing light on the consequences of cancer and its treatment. London: Macmillan, 2013. www.macmillan. org.uk/documents/aboutus/research/researchandevaluationreports/ throwinglightontheconsequencesofcanceranditstreatment.pdf 